\title{
Aperfeiçoando o aprendizado da Libras utilizando elementos de Internet das Coisas
}

\author{
Rodrigo Rozendo Bastos ${ }^{1}$, Lucas Lins ${ }^{1}$, Rafael de Amorim Silva ${ }^{2}$ \\ ${ }^{1}$ Instituto de Computação - Universidade Federal de Alagoas (UFAL) \\ Caixa Postal 61 - 57.300.970 - Maceió - AL - Brazil \\ $\{$ rrb, 1ll, rafael\}@ic.ufal.br
}

\begin{abstract}
The Internet of things has features and operation differentiated from traditional networks, being a potential technology for usage in various areas of the educational context. Based on that technology, this article proposes to build a system computational established among heterogeneous objects to improve the apprentice learning process for Brazilian Sign Language and to promote social inclusion of brazilian deaf.
\end{abstract}

Resumo. A Internet das Coisas possui características e funcionamento diferenciados das redes tradicionais, sendo uma potencial tecnologia para uso em diversas áreas do âmbito educacional. Baseado nessa tecnologia, este artigo propõe a construção de um sistema computacional estabelecido por objetos heterogêneos para aperfeiçoar o processo de ensino-aprendizagem da Língua Brasileira de Sinais e promover a inclusão social dos surdos brasileiros.

\section{Introdução}

Segundo o censo realizado em 2010 pelo Instituto Brasileiro de Geografia e Estatística (IBGE), 9,8 milhões de brasileiros possuem deficiência auditiva, o que representa 5,2\% da população brasileira. Deste total 2,6 milhões são surdos e 7,2 milhões apresentam grande dificuldade para ouvir (BRASIL, 2010).

A língua materna do surdo brasileiro é a Língua Brasileira de Sinais (Libras), que utiliza um canal viso-gestual, ou seja, articula-se por gestos e é percebida pela visão, enquanto a língua portuguesa, língua oficial para os ouvintes, se sustenta no canal oral auditivo, ou seja articula-se por via oral e é percebida pela audição. Este fato juntamente com as divergências sintáticas de ambas as línguas, trazem várias consequências, como por exemplo a exclusão social do surdo.

O aprendizado em Libras, tanto para o surdo quanto para ouvinte, é de grande importância para promover a inclusão social do indivíduo surdo. O processo de ensino deve ser iniciado durante a infância pois através da língua nos constituímos plenamente como seres humanos, nos comunicamos com nossos semelhantes, construímos nossas identidades e subjetividades, adquirimos e compartilhamos informações que nos possibilitam compreender o mundo que nos cerca (GESSER, 2009). 
Nos últimos anos, há uma forte demanda no setor de educação por tecnologias de informação e comunicação que propiciem uma tomada de decisão mais acurada e que estabeleçam um processo de ensino-aprendizagem mais eficiente. Tecnologias como sistemas tutores inteligentes (STI), automação em salas de aulas (SmartClass), ambientes virtuais de aprendizado (AVA), ferramentas colaborativas, Internet das Coisas (IoT) são exemplos de ferramentas que podem auxiliar professores e estudantes a obterem melhores desempenhos em seus processos educativos.

Neste contexto, este artigo descreve um sistema computacional para aperfeiçoar o aprendizado em Libras. O sistema consiste no uso de dispositivos vestíveis para captar a movimentação das mãos e dos braços do estudante para associar com um determinado sinal da Libras armazenado em nossa base de conhecimento.

O restante do artigo está estruturado como segue. A seção 2 apresenta alguns fundamentos relacionados a Libras, Captura de Movimento e Internet das Coisas. A seção 3 apresenta alguns trabalhos relacionados com a nossa aplicação. A nossa proposta é apresentada na Seção 4. Finalmente, a Seção 5 apresenta as considerações finais e direcionamentos para trabalhos futuros.

\section{Fundamentação Teórica}

\subsection{Língua Brasileira de Sinais (Libras)}

Em 24 de abril de 2002, a Lei $\mathrm{N}^{0} 10.436$, conhecida popularmente como Lei de Libras foi sancionada pelo então Presidente da República Fernando Henrique Cardoso (BRASIL, 2002) e determinada que:

"Entende-se como Língua Brasileira de Sinas - Libras a forma de comunicação e expressão, em que o sistema linguístico de natureza visual motora com estrutura gramatical própria, constituem um sistema linguístico de transmissão de idéias e fatos oriundos de comunidades de pessoas surdas do Brasil.” (BRASIL, 2002)

A Libras é a língua utilizada pela comunidade surda brasileira. Esta não é o português sinalizado, por ter estrutura própria, com regras próprias, a maior evidência disso é que existem surdos que se comunicam muito bem mas não sabem ler português. E é uma língua natural, por refletir valores culturais e por estar suscetível às mudanças impostas por comunidades e grupos sociais que utilizam. Ela não é universal. Assim como a língua portuguesa é falada no Brasil e a língua inglesa é falada nos Estados Unidos da América, que por sua vez tem a American Sign Language (ASL) como a língua utilizada pela comunidade surda americana. A Libras não é mímica, por possuir regras sintáticas, por apresentar regionalismos e, principalmente, por ser capaz de representar ideias abstratas. As ideias são expressas por sinais que são compostos por cinco parâmetros (KOJIMA, 2008):

(a) Configuração de mãos - forma da(s) mão(s) para fazer o sinal;

(b) Ponto de articulação - lugar no espaço ou parte do corpo no qual incide(m) a(s) mão(s);

(c) Direcionalidade - movimento que expressa ponto inicial e final ou origem e destino para o sinal;

(d) Movimento - movimento efetuado pela(s) mão(s); 
V Congresso Brasileiro de Informática na Educação (CBIE 2016)

Anais dos Workshops do V Congresso Brasileiro de Informática na Educação (CBIE 2016)

(e) Expressão Facial/Corporal - são expressões ou movimentos feitos com o corpo.

No escopo deste artigo iremos abordar e aplicar o sistema com base nos parâmetros: configuração de mão, movimento, direcionalidade e ponto de articulação.

\subsection{Captura de Movimento}

A captura de movimento de uma pessoa ou objeto pode ser feito a partir de diferentes tipos de sensores, os quais podem adotar tecnologias diferentes. Dentre os tipos de sensores de captação de movimento estão os: acústicos, eletromecânicos, magnéticos, baseados na emissão de sinais infravermelho, etc.

Nos sistemas acústicos, transdutores de ultra-som são utilizados na captura dos movimentos e fornecimento dos dados desejados. Nesses sistemas, emissores emitem sons captados pelos sensores. Um exemplo de aplicação desses sistemas pode ser visto nos carros, que os utilizam para alertar ao motorista de algum obstáculo quando este estiver dando ré no carro. Nos sistemas eletromecânicos geralmente é utilizado potenciômetros, que são colocados nas articulações de uma estrutura mecânica fixada no corpo de uma pessoa, que com o movimento do indivíduo, os sensores fornecem as posições angulares de cada articulação que está sendo analizada. Os sistemas que utilizam sinais infravermelhos podem oferecer o menor custo dentre as opções de sensores, porém alguns movimentos podem ficar ocultos ou serem erroneamente interpretados (SISTO, 2014)

\subsection{Internet das Coisas (IoT)}

A miniaturização e o barateamento dos mecanismos de comunicação como RFID, sensores de rede, NFC e redes de comunicação sem fio, aliados ao aumento da capacidade de processamento dos dispositivos embarcados, tem tornado o paradigma IoT cada vez mais palpável e aplicável a diferentes cenários. Este paradigma é baseado na troca de informações entre objetos físicos do mundo real, sendo composto por elementos sensoriais, conectividade e semântica.

O termo Internet of Things (IoT) foi cunhado originalmente em 1999 por Kevin Ashton, ao descrever um sistema no qual dispositivos eletrônicos com conectividade entre si são anexados aos objetos do mundo físico, utilizando sensores para captar informações deste mundo. Estes dispositivos do mundo físico se integram em um ambiente conectado no qual são capazes de serem identificados, possuem conectividade, externam seus estados e, eventualmente, possuem capacidade de processamento de tal modo a influenciar outros objetos, tanto virtuais quanto físicos. Portanto, tais objetos teriam a capacidade de trocar informações entre si e serem controlados remotamente. A crescente demanda por este tipo de dispositivo tem despertado o interesse da indústria e das instituições acadêmicas em prover tecnologias compatíveis com o conceito IoT. Segundo (AL-FUQAHA, 2015), estima-se que o número de objetos conectados na Internet no ano de 2010 tenha superado o da população da terra, alcançando cerca de 212 bilhões de dispositivos e representando 45\% do tráfico da Internet. Em termos de impacto financeiro, os autores em (Manyika, 2015) especulam que em 2025 o impacto econômico alcance 11,1 trilhões de dólares americanos. 
V Congresso Brasileiro de Informática na Educação (CBIE 2016)

Anais dos Workshops do V Congresso Brasileiro de Informática na Educação (CBIE 2016)

A pesquisa na área de IoT ainda não convergiu para um modelo geral de arquitetura de rede. As propostas existentes na literatura definem modelos similares aos encontrados na arquitetura TCP/IP, embora com funcionalidades distintas em cada camada. Os modelos arquiteturais para IoT ilustrados na Figura 1 contém similaridades em suas camadas em relação ao TCP/IP, mas se diferenciam nas camadas inferiores, abstraindo o processo de comunicação dos objetos físicos, e nas camadas superiores, voltando-se para o provimento de serviços.
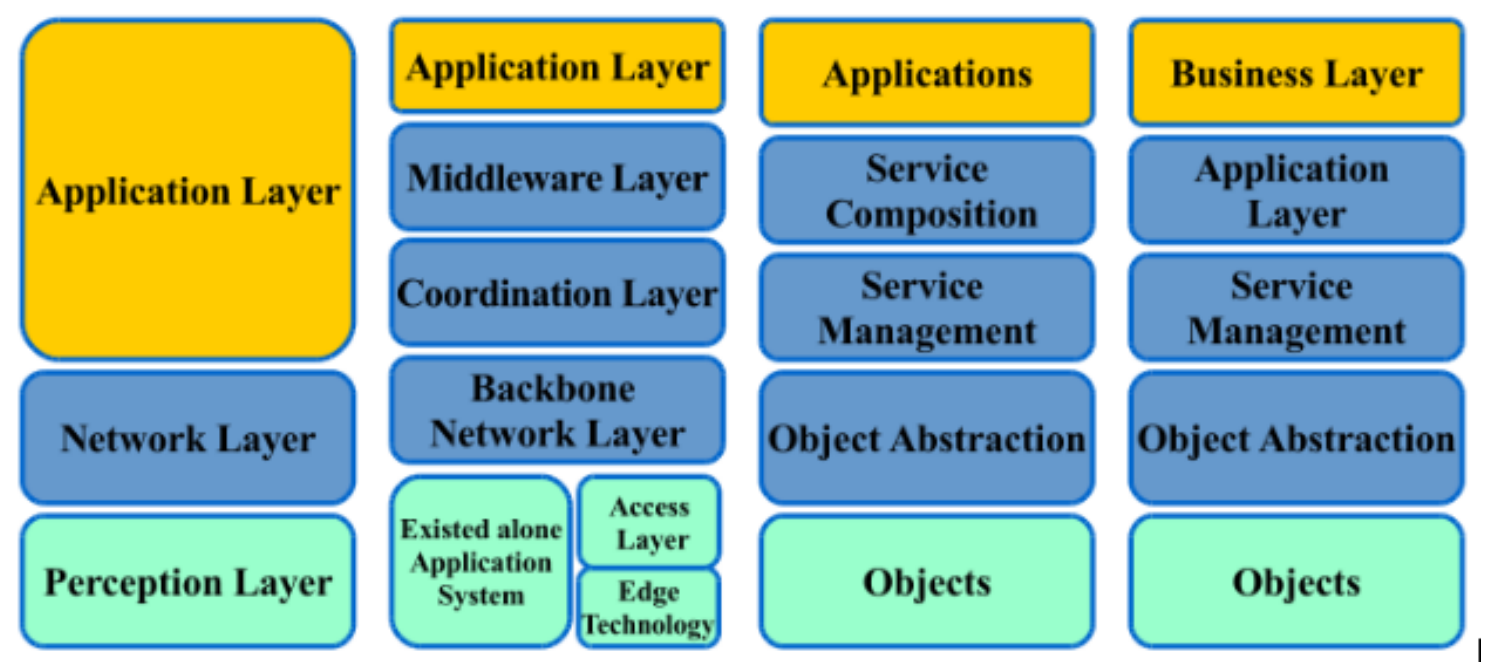

Figura 1: Propostas de modelos arquiteturais para o paradigma de IoT (AL-FUQAHA, 2015)

\section{Trabalhos Relacionados}

O projeto apresentado neste artigo faz parte da área de pesquisa conhecida como reconhecimento de língua de sinais com uso de software. No estado da arte existem diversas abordagens, sendo classificadas nos seguintes grupos: (i) reconhecimento de Libras utilizando câmeras de vídeo; (ii) reconhecimento de Libras utilizando dispositivos sensoriais (wearables) ou sensores de captura de vídeo e profundidade da cena; ou (iii) reconhecimento de Libras utilizando jogos WEB.

No grupo (i), destacam-se os projetos SensorLibras e Giulia. O projeto SensorLibras é baseado no uso de luvas sensoriais, utilizando um dispositivo Sun SPOT para ler dados capturados pelos sensores que serão utilizados no processo de captação dos movimentos das mãos no espaço tridimensional. Além do reconhecimento do sinal gesticulado, o SensorLibras realiza a tradução para Língua Portuguesa (TAVARES, 2010). Por outro lado, o projeto Giulia utiliza a braçadeira Myo, ao invés de luvas, para realizar a captura do movimento dos sinais feitos pelo usuário. O Myo, através do contato com a pele, captura a atividade elétrica dos músculos do braço e em seguida estabelece uma comunicação via bluetooth com um aplicativo desenvolvido para plataforma android. Assim como SensorLibras, o aplicativo Giulia realiza a tradução da Libras para o português com o uso de técnicas de Inteligência Artificial (MAP, 2015). 
No grupo (ii), destacam-se os projetos definidos em TEODORO (2014) e em PORFIRIO (2013). Os autores em TEODORO (2014) segue uma abordagem diferente em comparação com os trabalhos relacionados do grupo (i), pois, neste caso, o reconhecimento de Língua Brasileira de Sinais é realizado através do processamento de imagens e vídeos digitais. O software é treinado e testado a partir de uma base de conhecimento com vídeos e imagens de pessoas se comunicando em Libras. Em contrapartida, o projeto definido em PORFIRIO (2013) utiliza o sensor de movimentos Kinect da Microsoft para desempenhar reconhecimentos de 61 diferentes configurações gestuais da mão utilizando um método de classificação de malhas 3D da mão. Tais autores relatam uma acurácia de até 93\% no processo de reconhecimento gestual.

No grupo (iii) destaca-se o jogo Leap-Libras, definido em (LEAPEC, 2016). Este jogo oferece uma interface gráfica WEB para que o usuário com deficiência auditiva possa praticar o aprendizado em Libras.

O diferencial da nossa proposta em relação aos trabalhos apresentados anteriormente é a utilização de luvas sensoriais para captura dos sinais gesticulados a partir de determinados parâmetros, da Libras, da configuração de mão, do ponto de articulação, do movimento e da direcionalidade. Além disso, os outros trabalham enfatizam o processo de tradução da língua de sinais Libras, enquanto que o nosso trabalho enfatiza o uso de um tutor inteligente para ensinar como desempenhar o sinal de Libras, além de oferecer o serviço de tradução.

\section{Proposta}

Esta seção descreve um sistema computacional para o ensino eficiente dos sinais da Libras. Tal sistema é composto por um conjunto de objetos físicos que possuem a capacidade de se comunicarem através de uma rede IoT para entregar uma experiência mais rica ao usuário neste processo de ensino. O sistema utiliza tecnologias baseadas na computação vestível para captar a movimentação dos dedos e dos braços para associar com um determinado padrão armazenado em um banco de dados distribuído. Esta associação indicará qual sinal o usuário está realizando em um dado momento e se tal sinal está sendo executado de forma adequada para a representação destes sinais. A Figura 2 apresenta o diagrama com os módulos da aplicação do sistema computacional proposto. 
V Congresso Brasileiro de Informática na Educação (CBIE 2016)

Anais dos Workshops do V Congresso Brasileiro de Informática na Educação (CBIE 2016)

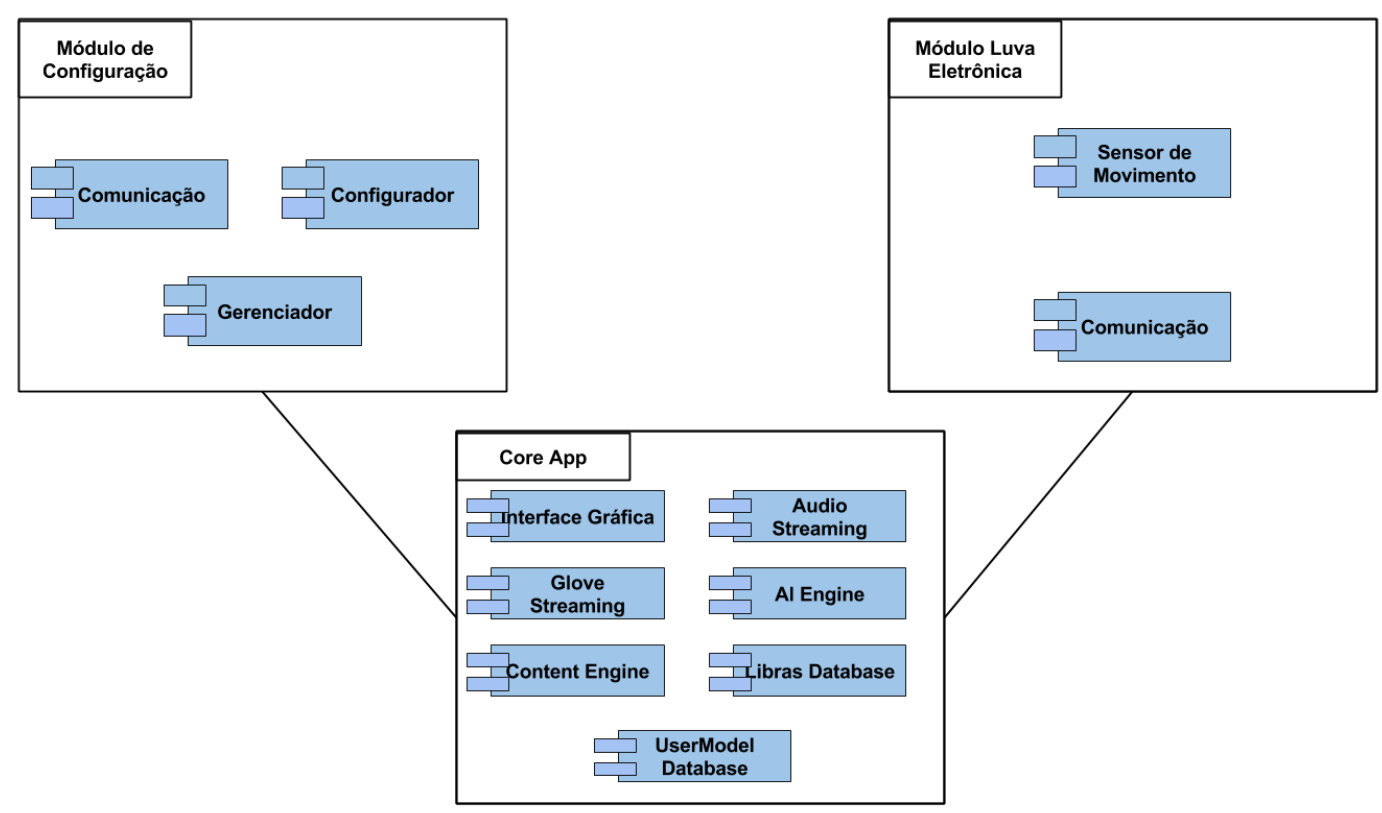

Figura 2: Diagrama de Módulos da Aplicação

O módulo Luva Eletrônica adota a luva CyberGlove III, fabricada pela CyBerGlove Systems. Esta luva possui até 22 sensores de alta precisão, sendo capaz de captar toda a movimentação, altura e posição das mãos para a interpretação de sinais pela aplicação principal. Tal luva possui as seguintes características: (i) armazenamento de dados portável; (ii) compatibilidade com sensores de alta precisão; (iii) permite a montagem de marcadores de câmera na luva para rastreamento; (iv) conexão WiFI, USB e SD Card; (v) compatibilidade com aplicações antigas; e (vi) produz dados adicionais da mão para animações mais realísticas.

O módulo Core App contém a interface gráfica da aplicação, que poderá ser rodada em qualquer sistema operacional Android. Tal aplicação pode ser executada, por exemplo, em hardwares de óculos virtuais, tablets, ou smartphones. O banco de dados utilizado deve ser orientado a objetos (e.g. NoSQL). A máquina de processamento da inteligência artificial deve ser baseada em algoritmo de reconhecimento de padrões (e.g. redes neurais ou lógica fuzzy, dependendo do contexto) e a streaming de audio deve ser processada para ser enviada a uma headset com WiFi ou Bluetooth.

Por último, o módulo de configuração é composto por um gerenciador, responsável em alterar as configurações da aplicação principal, além de um comunicador que utiliza o padrão WiFi para realizar tal comunicação.

\subsection{Funcionalidades (Casos de Uso)}

Sinais da Língua Brasileira de Sinais serão apresentados em uma interface gráfica e o usuário deverá representar cada sinal utilizando gestos em sua mão, na altura e na sequência adequada, para que o sistema indique se o usuário de fato aprendeu o sinal. $\mathrm{O}$ usuário também poderá representar uma determinada palavra no idioma português em sua respectiva Libras. Uma sequência de Libras poderá ser apresentada para representar palavras e frases. 
V Congresso Brasileiro de Informática na Educação (CBIE 2016)

Anais dos Workshops do V Congresso Brasileiro de Informática na Educação (CBIE 2016)

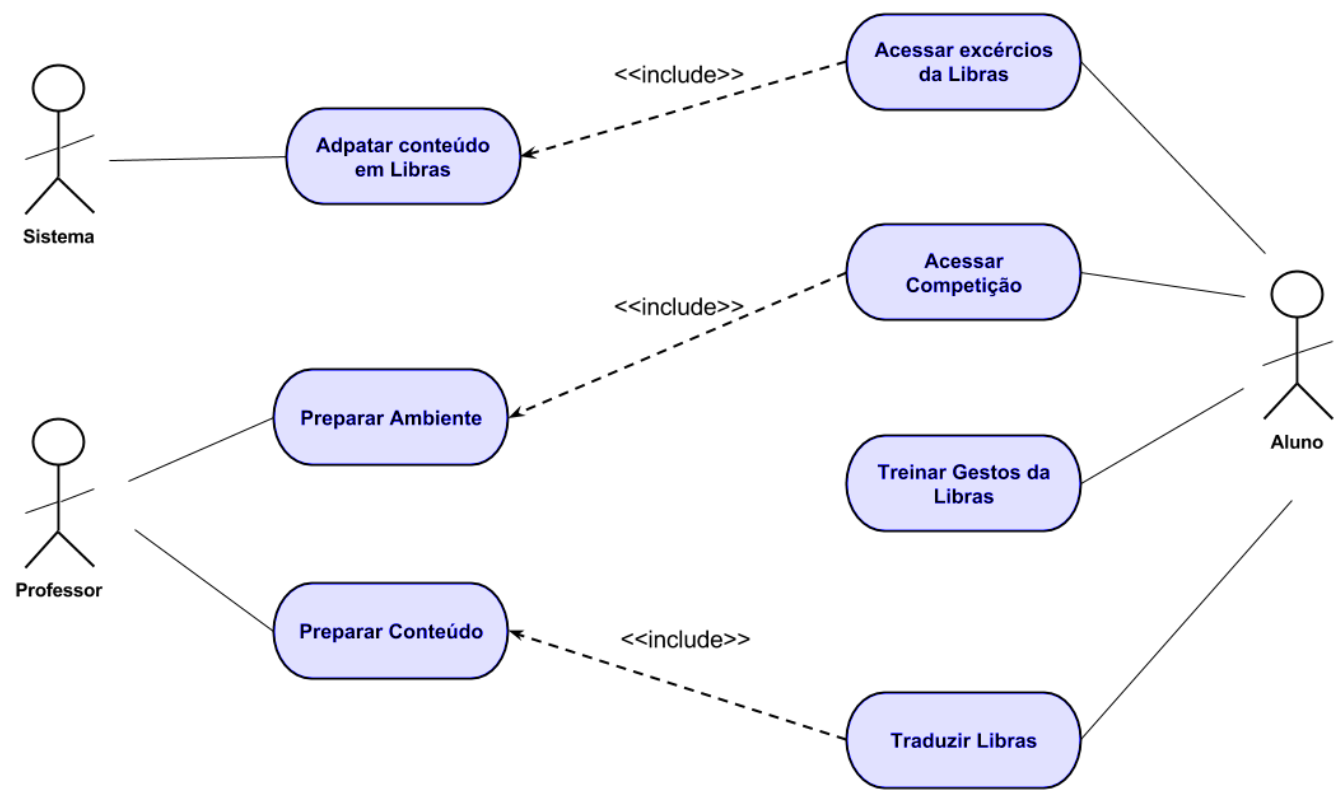

Figura 3: Diagrama de casos de uso da solução proposta

A Figura 3 apresenta as funcionalidades da solução proposta através de um diagrama de casos de uso. As seguintes funcionalidades são descritas a seguir:

- Acessar Exercício de Libras - a partir desta funcionalidade o usuário (estudante) irá receber uma lista de exercício, de forma sequencial, com o objetivo de aprimorar os seus conhecimentos na Língua Brasileira de Sinais.

- Acessar Competição de Libras - o objetivo desta funcionalidade é estimular o aprendizado do estudante através da gamificação do ensino.

- Treinar gestos da Libras: o usuário irá selecionar esta opção no menu principal e poderá buscar/selecionar em nosso dicionário uma determinada palavra, em português, para treinar sua gesticulação em Libras.

- Traduzir Libras: o estudante poderá realizar a tradução da libras para língua portuguesa com esta funcionalidade. Ele irá realizar a sequência de sinais em Libras e o sistema irá retornar a tradução, em forma de texto, em português.

- Adaptar conteúdo: o sistema pode adaptar o conteúdo dos exercícios em Libras de acordo com a performance do usuário com o ambiente

- Preparar ambiente: o professor tem a possibilidade de preparar o ambiente adequado para cada estudante. Ele pode 
V Congresso Brasileiro de Informática na Educação (CBIE 2016)

Anais dos Workshops do V Congresso Brasileiro de Informática na Educação (CBIE 2016)

- Preparar conteúdo: o professor tem a possibilidade de gerar um conteúdo específico para o aprendizado do estudante.

\subsection{Arquitetura}

A Figura 4 ilustra a arquitetura de rede proposta neste trabalho. Esta arquitetura consiste de enlaces com o padrão IEEE 802.11 (WiFi) e IEEE 802.15.1 (BlueTooth). O restante das camadas utiliza a arquitetura TCP/IP convencional, exceto a luva eletrônica, consistindo na Figura 1, sendo o primeiro modelo de referência, da esquerda para a direita. Este modelo é o mais simples de todos os modelos apresentados nesta figura, contendo apenas a camada de aplicação, a camada de rede e a camada de percepção, responsável pela captura dos dados advindos dos sensores associados aos objetos desta proposta.
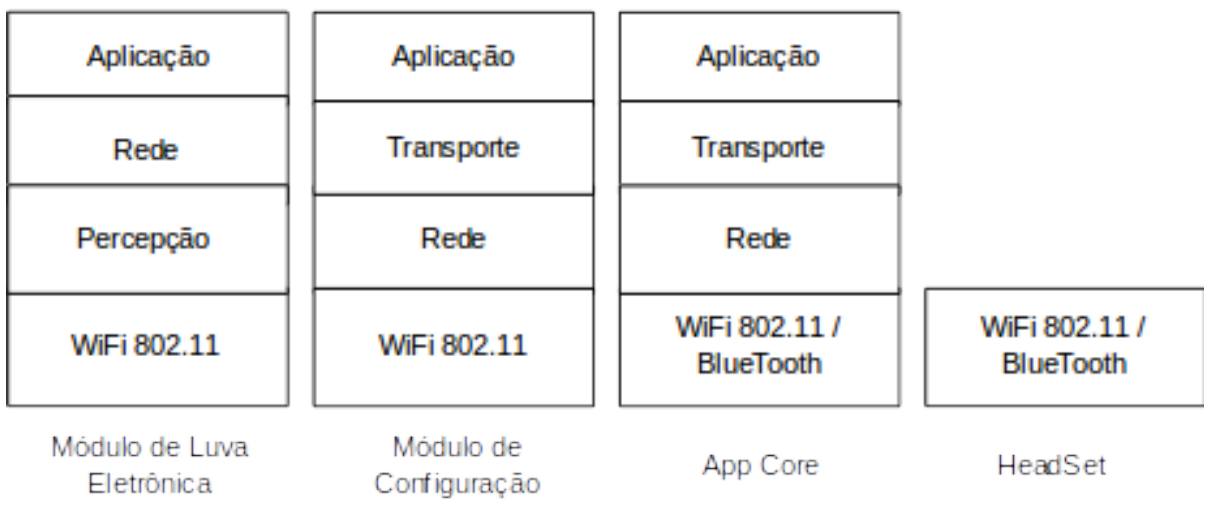

Figura 4: Arquitetura de Rede Proposta

A escolha deste modelo é devido aos seguintes fatores: (i) Devido a exigência de se rapidamente adquirir dados relativos a movimentação da luva, processá-los e utilizálos para a interface gráfica desta proposta, a arquitetura deverá ter camadas básicas para um rápido processamento; (ii) A abordagem proposta deve ser sensível ao atraso, exigindo um alto nível de processamento e resposta (em tempo real) para que a experiência do usuário no aprendizado de LIBRAS seja efetiva e eficiente; (iii) Das arquiteturas ilustradas na Figura 1, esta arquitetura é a mais eficiente na instalação e no gerenciamento dos pacotes inseridos na rede.

\subsection{Sistema IoT para o ensino da Libras}

O sistema proposto incorpora os seguintes elementos da Internet das coisas: (i) sensoriamento; (ii) semântica; (iii) dados. 
V Congresso Brasileiro de Informática na Educação (CBIE 2016)

Anais dos Workshops do V Congresso Brasileiro de Informática na Educação (CBIE 2016)

No sensoriamento, realizaremos a captura do movimento através do uso da computação vestível. A computação vestível é uma abordagem que tem como objetivo tornar os computadores parte do nosso dia-a-dia, incorporando-os em roupas, sapatos ou relógios, óculos e etc. Em nosso sistema computacional iremos utilizar luvas de sensoriamento, com a função de capturar os movimentos dos braços e mãos que ocorrem durante as gesticulações em Libras, realizadas pelo usuário. Os dados dos movimentos capturados serão passados para a nossa aplicação que será executada em outro dispositivo vestível, um óculos (SmartGlass). Nos elementos de semântica e dados, será realizado o processo de extração de conhecimento. O objetivo desse processo é reconhecer e analisar os dados para realizar associações coerentes e fornecer o resultado adequado para o usuário. O processo de reconhecimento será implementado com uso de algoritmos de inteligência artificial. Podemos observar o fluxo descrito acima através do Diagrama de Estados apresentado na Figura 4.

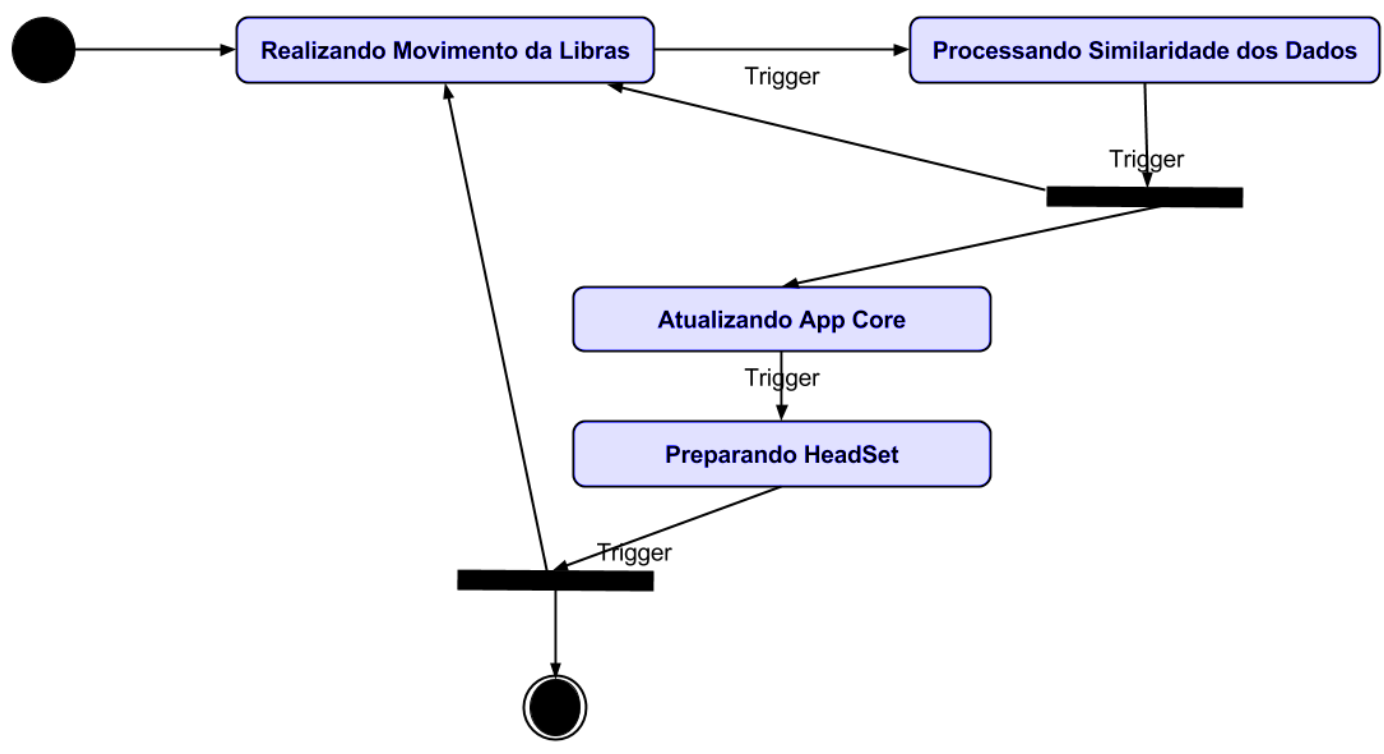

Figura 5: Diagrama de Estados

\section{Considerações finais}

Este trabalho apresentou uma proposta de sistema computacional utilizando elementos da Internet das Coisas para aperfeiçoar o aprendizado em Libras. O objetivo do trabalho é proporcionar a inclusão social dos surdos através do ensino-aprendizagem de Libras com uso da tecnologia.

Como trabalhos futuros, iremos iniciar o processo de desenvolvimento da ferramenta proposta, essa fase contará com o implementação do software responsável por realizar associação adequada dos sinais em Libras e a construção dos dispositivos vestíveis para o sensoriamento dos movimentos do usuário. 
V Congresso Brasileiro de Informática na Educação (CBIE 2016)

Anais dos Workshops do V Congresso Brasileiro de Informática na Educação (CBIE 2016)

\section{Referências}

AL-FUQAHA, A. et al. Internet of things: A survey on enabling technologies, protocols, and applications. Communications Surveys \& Tutorials, IEEE, IEEE, v. 17, n. 4, p. 2347-2376, 2015.

BBRASIL. Lei no 10.436, de 24 de abril de 2002. Lei de Libras. Diário Oficial de República Federativa do Brasil, Brasília, DF.

BRASIL. Ministério do Planejamento, Orçamento e Gestão. Instituto Brasileiro de Geografia e Estatística. Censo Demográfico 2010. Disponivel em: http://censo2010.ibge.gov.br/apps/atlas/pdf/Pag_203_Religi\%C3\%A3o_Evang_miss \%C3\%A3o_Evang_pentecostal_Evang_nao\%20determinada_Diversidade \%20cultural.pdf. Acesso em: agosto de 2016.

KOJIMA, C. K. LIBRAS: Línguas Brasileira de Sinais: a imagem do pensamento. São Paulo: Editora Escala, v. I, II, III, IV \& V, 2008.

LEAPC. Leapc Libras. Disponível em: https://leaplibras.herokuapp.com/. Acesso em: Agosto de 2016.

MAP. Projeto Giulia "A mão que fala"- Formulário de Aquisição. Disponvível em: http://maptechnology.com.br/pt-br/novidades/projeto-giulia-m\%C3\%A3o-que-falaformul\%C3\%A1rio-de-aquisi\%C3\%A7\%C3\%A3o. Acesso em: agosto de 2016.

PORFIRIO, A. J., Wiggers, K. L., Oliveira, L. E. S. and Weingaertner, D. LIBRAS Sign Language Hand Configuration Recognition Based on 3D Meshes. IEEE International Conference on Systems, Man, and Cybernetics. pp.:1588 - 1593. 2013.

SISTO, T. M. e Cabral, E. L. L. Sistema de Captura de Movimento com Sensores Infravermelho. 2014

TAVARES, João E. da R. SensorLibras: Tradução Automática Libras-Português através da computação ubiqua. In: II Congresso Nacional de Pesquisas em Tradução e Interpretação de Libras e Língua Portuguesa, Universidade Federal de Santa Catarina - UFSC, Novembro, 2010.

TEODORO, B. and Digiampietri, L. A. Desenvolvimento de um Sistema de Reconhecimento Automático de Língua Brasileira de Sinais. In: VII Workshop de Teses e Dissertações em Sistema de Informação, maio, 2014 